\title{
Abbreviations and notations
}

$\begin{array}{ll}\text { a.s. } & \begin{array}{l}\text { Almost surely } \\ \text { CLT }\end{array} \\ \stackrel{\text { Central limit theorem }}{=} & \begin{array}{l}\text { Equality in distribution } \\ \text { the space of càdlàg functions defined on }[0, T], \text { i.e., functions continuous } \\ \text { from the right and with the left limits }\end{array} \\ \mathrm{fBm} & \text { Fractional Brownian motion } \\ \mathrm{r} . \mathrm{v} . & \text { Random variable } \\ \mathrm{EMM} & \text { Equivalent martingale measure } \\ \mathrm{ELMM} & \text { Equivalent local martingale measure } \\ \stackrel{\mathrm{d}}{\rightarrow} & \text { Weak convergence in distribution of the random variables } \\ \stackrel{\mathbb{P}}{\rightarrow} & \text { Convergence in probability of the random variables } \\ \stackrel{\mathbb{Q}^{(n)}, \mathbb{Q}, d}{\longrightarrow} & \text { Weak convergence in distribution of the random variables, where the }\end{array}$

measures $\mathbb{Q}^{(n)}$ and $\mathbb{Q}$ correspond to the pre-limit r. v. and the limit r. v., respectively

$\stackrel{\mathbb{Q}^{(n)}, \mathbb{Q} \text {, fdd }}{\Longrightarrow}$ Weak convergence of finite-dimensional distributions of the stochastic processes

$\mathbb{Q} \sim \mathbb{P} \quad$ Measures $\mathbb{Q}$ and $\mathbb{P}$ are equivalent

$\stackrel{\mathbb{Q}^{(n)}, \mathbb{Q}}{\longrightarrow} \quad$ Weak convergence of the measures corresponding to the stochastic processes

$\lfloor a\rfloor \quad$ The biggest integer number not exceeding $a$

$\lceil a\rceil$ The fractional part of $a$, i. e., the difference $a-\lfloor a\rfloor$

$\operatorname{Bi}_{\mathrm{rt}}(L, l, p)$ The right-tailed binomial distribution, i. e., the probability that at least $l$ successes occur in $L$ Bernoulli trials with parameter $p$

$\Phi(x) \quad=\frac{1}{\sqrt{2 \pi}} \int_{-\infty}^{x} e^{-y^{2} / 2} d y$, the standard normal distribution function (standard normal cumulative distribution function, standard Gaussian distribution function)

$\left(\begin{array}{l}n \\ k\end{array}\right) \quad=\frac{n !}{k !(n-k) !}$, binomial coefficient

$\left.\frac{d \mathbb{Q}}{d \mathbb{P}}\right|_{\mathcal{F}_{t}} \quad$ The restriction of the Radon-Nikodym derivative $\frac{d \mathbb{Q}}{d \mathbb{P}}$ on the $\sigma$-field $\mathcal{F}_{t}$

i. i. d. Independent identically distributed

i. i.d.r.v. Independent identically distributed random variables

$a \cdot b \quad$ Inner product in any finite-dimensional space

$\mathbb{C} \quad$ Call option and/or any European contingent claim that is not specified

$\mathbb{P} \quad$ Put option

$\pi_{t}^{\text {call }}(x) \quad$ Fair (non-arbitrage, calculated with respect to the unique martingale measure) option price of a discounted call option as the function of the initial price $x$ of the underlying asset, with intermediate expiry date $t \in[0, T]$ and calculated at zero moment of time in the complete market 
$\pi_{t}^{\text {put }}(x) \quad$ Fair (non-arbitrage, calculated with respect to the unique martingale measure) option price of a discounted put option as the function of the initial price $x$ of the underlying asset, with intermediate expiry date $t \in[0, T]$ and calculated at zero moment of time in the complete market $\pi^{\text {call }}(x) \quad=\pi_{T}^{\text {call }}(x)$ if the market is considered at the time interval $[0, T]$ and $T$ is an ultimate expiry date

$\pi^{\text {put }}(x) \quad=\pi_{T}^{\text {put }}(x)$ if the market is considered at the time interval $[0, T]$ and $T$ is an ultimate maturity date

$\mathbb{T}$ The time set on which the financial market is considered. It can be a finite, countable set, some interval, or $\mathbb{R}^{+}$.

$\mathbb{Z}^{+} \quad 0,1,2, \ldots$ 